\title{
Centre romand des brûlés, filière commune enfant et adulte
}

\author{
Anthony de Buys Roessingh, \\ Christelle Jung, \\ Wassim Raffoul, \\ Lee Laurent-Applegate, \\ Jacqueline Jeanmonod, \\ Mette Berger
}

Pour la «filière brûlé» du CHUV
Le Centre Romand des Grands Brûlés (CRB) est l'un des deux centres suisses spécialisés dans le traitement des victimes de brûlures graves. Des équipes multidisciplinaires, à la fois médicales, chirurgicales et soignantes s'occupent de ces blessés 24 heures sur 24 et 7 jours sur 7 . Chaque année $150-200$ patients bénéficient des traitements au CRB. Ce dernier comporte 4-5 lits de soins intensifs adultes intégrés dans un service de médecine intensive multidisciplinaire. Les enfants gravement brûlés sont traités dans les soins intensifs de pédiatrie (2 lits). A ce jour, on compte de nombreux avantages à l'immersion des grands brûlés au sein des soins intensifs comme cela est organisé au CHUV: suivi médical intensif de niveau universitaire incluant toutes les techniques d'assistance spécifiques, collaboration médicochirurgicale directe, développement des compétences infirmières, formation continue, fidélisation et valorisation des soignants. Un bloc opératoire et une salle de douche, destinés aux adultes et aux enfants, sont intégrés dans le centre.
- de poursuivre un programme de recherche original dans le domaine de la réanimation, de la chirurgie et des soins aux brûlés.

La prise en charge à la sortie des soins intensifs se déroule dans le service de chirurgie plastique reconstructive et de la main pour les adultes et dans le service de chirurgie pédiatrique pour les enfants, avec le soutien logistiques des adultes pour les brûlés enfants de plus de $20 \%$ de la surface corporelle.

Ainsi, l'élaboration d'une filière commune adulte et enfant s'inscrit dans la reconnaissance politique de la prise en charge des grands brûlés dans le cadre de la médecine hautement spécialisée (MHS) [4, 5]. Sa réalisation au CHUV en 2011 tenait compte des particularités de la prise en charge des grands brûlés, notamment une activité hautement spécialisée, des urgences lourdes, des coûts importants et une multidisciplinarité fondamentale pour une prise en charge adéquate. Elle a impliqué la mise en relation de l'ensemble des protagonistes en lien avec la brû-

\section{«Les durées d'hospitalisation sont limitées au strict minimum, afin que les enfants puissent réintégrer au plus vite leur famille.»}

Correspondance:

PD Dr. Anthony de Buys Roessingh

Service de chirurgie pédiatrique CHUV-BH10

CH-1011 Lausanne

www.chuv.ch/brulures/ brul_home/
Le bénéfice d'une prise en charge des grands brûlés au sein de centres spécialisés est établi et fait l'objet d'un consensus depuis les années 1970 [1-3]. Les brûlures profondes sont parmi les traumatismes les plus lourds en termes de besoins de soins, de ressources humaines et de plateau technique.

Les missions du CRB sont:

- de prendre en charge tous les patients brûlés des cantons romands et parfois de Suisse alémanique lorsque le centre zurichois est surchargé. Dans la mesure des disponibilités, des patients de France voisine y sont également soignés;

- de prodiguer des traitements de qualité en mettant à disposition une équipe pluridisciplinaire et un plateau technique spécialisé dans des structures adaptées;

- d'assurer la formation et l'enseignement dans les spécialités requises pour la prise en charge des brûlés depuis le préhospitalier jusqu'à la réhabilitation; lure afin de garantir une prise en charge cohérente et une continuité des soins depuis le lieu de l'accident jusqu'au retour à domicile, sans oublier le suivi ambulatoire. L'établissement d'itinéraires cliniques s'inscrit dans une démarche de qualité médicale en visant à satisfaire les besoins du malade tout en mesurant les résultats effectifs et les coûts induits par les soins. Il s'agit de replacer le patient au centre du système de soin, en organisant une prise en charge coordonnée en fonction de ses besoins [6].

En ce qui concerne les enfants plus spécifiquement, les accidents domestiques sont courants: $80 \%$ des brûlures surviennent à domicile aux heures des repas et en présence de parents [7]. Nous avons à Lausanne, par année, 250 urgences de brûlures chez l'enfant, 280 soins sous narcose pour débridements et pansement, et 30 interventions chirurgicales avec greffe de peau. Les durées d'hospitalisation sont limitées au strict minimum, afin que les enfants puissent réintégrer au plus vite leur famille. L'in- 
frastructure lausannoise, caractérisée par cette prise en charge commune des enfants avec les adultes, constitue un partage des connaissances tant au point de vue de l'anesthésie, des soins intensifs, des soins infirmiers, de la nutrition, des traitements de physiothérapie et d'ergothérapie, du suivi psychologique que des aspects chirurgicaux. Une brochure informative, établie par toutes les équipes intervenant dans cette prise en charge, a été conçue pour les enfants et leurs proches. Elle facilite ainsi la compréhension des différentes étapes de cette longue prise en charge.

La filière brûlés ne s'arrête pas à la sortie du CHUV mais intègre le parcours de vie après la brûlure. Le centre des brûlés collabore avec la clinique de réadaptation SUVA Care à Sion ainsi qu'avec l'association de patients brûlés FLAVIE. La Clinique Romande de Réadaptation SUVA Care inaugurée en 1999, est devenue un centre de référence en réadaptation des grands brûlés pour la Suisse Romande. FLAVIE (www.flavie.ch) en tant qu'association suisse romande pour les personnes brûlées et leur entourage, favorise la constitution de réseaux, influence les politiques de santé et développe la prévention. Elle offre du soutien et des conseils sur les plans sociaux, juridiques et financiers. Elle agit gratuitement en favorisant la réinsertion sociale (coaching thérapie régénérative du service de chirurgie reconstructive et de la main, le service de médecine intensive adulte et le service de chirurgie pédiatrique. De nombreux projets sont en cours, tant cliniques que fondamentaux.

$\mathrm{Au}$ niveau prévention, nous avons réalisé huit spots de prévention avec huit scénarios basés sur des accidents banals du quotidien chez l'enfant. Ces spots seront diffusés dès le 18 juin 2014 dans toute la Suisse. Au niveau formation, de nombreux cours sont organisés en interne (voir site web).

Autour du thème des enfants brûlés, nous organisons le 17 juin 2014 au CHUV (dès 13 h, Auditoire Mathias Mayor) une rencontre-débat sur le sujet: Prise en charge à long terme des enfants brûlés, chirurgie secondaire, maquillage et cure thermale, avec des équipes invitées de Paris. Inscription/Informations: Sarah.besson[at]chuv.ch (reconnu pour des crédits SSP).

En résumé, la «filière brûlé» est opérationnelle depuis janvier 2011. Elle a permis la mise en lien et a encouragé les échanges entre tous les intervenants de la chaîne de soin, entraînant une meilleure connaissance du rôle de chacun, une vision globale de la prise en charge et une amélioration de la prise en charge des patients.

\section{«La filière brûlés ne s'arrête pas à la sortie du CHUV mais intègre le parcours de vie après la brûlure.»}

personnel, activités de loisirs, parrainage par d'autres personnes accidentées, soutien pour les proches), en facilitant la réinsertion scolaire par une sensibilisation dans les écoles, en offrant un entretien conseil avec un avocat spécialisé ou un soutien financier ponctuel et en travaillant à l'amélioration de la prise en charge après la sortie de l'hôpital.

En ce qui concerne les cures thermales, celles-ci permettent d'atténuer le prurit, de calmer l'inflammation cicatricielle et d'assouplir les cicatrices. Leurs effets bénéfiques sont liés au bienfait de l'eau thermale elle-même et aux traitements de types massages de cicatrices et douches filiformes. Aucune structure d'accueil n'existe actuellement en Suisse romande pour accueillir les enfants brûlés. Ceci fait l'objet actuellement, par notre groupe, d'un travail de faisabilité et de création, en favorisant toujours le travail en commun des adultes et des enfants afin d'obtenir une masse critique adéquate.

$\mathrm{Au}$ niveau recherche, les équipes «adulte et enfant» travaillent en commun au sein de l'unité de recherche brûlé qui associe les différents services impliqués dans cette prise en charge, comme l'unité de

\section{Références}

1 Linn BS, Stephenson Jr. SE, Bergstresser PR, Smith J. Are burn units the best places to treat burn patients? J Surg Res. 1977;23(1):15.

2 Sheridan RL. Burn care: Results of technical and organizational progress. JAMA. 2003;290(6):719-22.

3 Berger MM, Raffoul W, Maravic P, Séchet V, Chioléro R. Pourquoi deux Centres de Brûlés adultes en Suisse, dont un au CHUV. Bull Méd Suisses. 2004;85(18):938-43.

4 CDS - Conference suisse des directrices et directeurs cantonaux de la sante: Liste des hôpitaux MHS. Disponible sur: www.gdk-cds.ch/index.php/ id $=983 \& \mathrm{~L}=1$

5 CDS - Conference suisse des directrices et directeurs cantonaux de la sante:Liste des hôpitaux MHS. Disponible sur: www.gdk-cds.ch/index.php/ $\mathrm{id}=903 \& \mathrm{~L}=1$

6 Vanhaecht K, Panella M, Zelm RV, Sermeus W. Is there a future for pathways? Five pieces of the puzzle. Int J Care Pathw. 2009;13(2):82-6.

7 Natterer J, de Buys Roessingh A, Reinberg O, Hohlfeld J. Targeting burn prevention in the pediatric population: a prospective study of children's burns in the Lausanne area. Swiss Medical Weekly. 2009;139:535-9. 